\title{
Chiral transition in a magnetic field and at finite baryon density
}

\author{
Jens O. Andersen* and Rashid Khan丹 \\ Department of Physics, Norwegian Institute of Science and Technology, N-7491 Trondheim, Norway
}

(Dated: September 24, 2018)

\begin{abstract}
We consider the quark-meson model with two quark flavors in a constant external magnetic field $B$ at finite temperature $T$ and finite baryon chemical potential $\mu_{B}$. We calculate the full renormalized effective potential to one-loop order in perturbation theory. We study the system in the large$N_{c}$ limit, where we treat the bosonic modes at tree level. It is shown that the system exhibits dynamical chiral symmetry breaking, i. e. that an arbitrarily weak magnetic field breaks chiral symmetry dynamically, in agreement with earlier calculations using the NJL model. We study the influence on the phase transition of the fermionic vacuum fluctuations. For strong magnetic fields, $|q B| \sim 5 m_{\pi}^{2}$ and in the chiral limit, the transition is first order in the entire $\mu_{B}-T$ plane if vacuum fluctuations are not included and second order if they are included. At the physical point, the transition is a crossover for $\mu_{B}=0$ with and without vacuum fluctuations.

PACS numbers: 11.15Bt, 04.25.Nx, 11.10Wx, 12.38Mh
\end{abstract}

\section{INTRODUCTION}

A tremendous amount of work has been done in recent years to map out the QCD phase diagram as a function of temperature $T$ and baryon chemical potential $\mu_{B}$. Two aspects have received particular attention: the position of the critical endpoint where the curve of first-order chiral transitions terminates in a second-order transition [1], and the various color-superconducting phases at large baryon chemical potential and low temperature [2].

With $N_{f}$ quark flavors, the global symmetry of QCD is $S U\left(N_{f}\right)_{V} \times S U\left(N_{f}\right)_{A}$ in the chiral limit and $S U\left(N_{f}\right)_{V}$ if the quark masses are equal. In the chiral limit with $N_{f}=2$, we use the isomorphism between the groups $S U(2) \times S U(2)$ and $O(4)$ and use the $O(4)$ linear sigma model (LSM) as a low-energy effective theory for QCD. Coupling the linear sigma model to quarks we obtain the quark-meson (QM) model. Since we have quark degrees of freedom, we can couple the model to a baryon chemical potential $\mu_{B}$ and study finite-density effects. The QM model has been used to study various aspects of the chiral transition at $\mu_{B}=0[3-7]$ and $\mu_{B} \neq 0[8-10]$. Schwinger-Dyson equations were used in [12]. One can couple the Polyakov loop to the quark sector by introducing a constant temporal gauge field background [13], in order to incorporate certain aspects of the deconfinement transition. The thermodynamics of the Polyakovextended quark-meson model (PQM) was studied in Refs. [14 22], and the PNJL model in Refs. [16, 23 29]

Using the QM model, it is a common approximation to omit the quantum and thermal fluctuations of the

\footnotetext{
*Electronic address: andersen@tf.phys.no

${ }^{\dagger}$ Electronic address: rashid.khan@ntnu.no
}

bosonic degrees of freedom, i.e. treating them at tree level. This is the large- $N_{c}$ limit. On the other hand, one keeps the thermal fluctuations of the quarks. Since symmetry breaking takes place in the mesonic sector and at tree level, the vacuum fluctuations of the quarks are sometimes omitted; however one must be careful when making such additional approximations. It was shown in Ref. 7] that the order of the phase transition depends on whether or not one includes the fermionic vacuum contributions in effective potential. This issue was studied in detail in [10] using optimized perturbation theory [11]. If all bosonic and fermionic vacuum and thermal contributions are included, resummed one-loop results predict a first-order transition in the entire $\mu-T$ plane in the chiral limit.

For $N_{f}=2$ and in the chiral limit, universality and renormalization arguments firmly establish the secondorder nature of the transition at zero baryon chemical potential if the axial $U(1)$ symmetry is explicitly broken. Otherwise it is driven first order. For $N_{f}=3$, the transition is always first order in the limit of zero current quark masses. [1, 30]. At the physical point, i. e. for current quark masses that yield $m_{\pi}=140 \mathrm{MeV}$, it is a crossover transition. At nonzero baryon chemical potential $\mu_{B}$ and in particular at $T=0$, the order of the chiral transition is not obvious from universality arguments [31]. Most model calculations predict a firstorder transition at $T=0$ [1, 32], although the results depend on the input parameters and are sensitive to the ultraviolet cutoff. If there is a first-order transition at $T=0$, there is a line of first order transitions in the $\mu_{B}-T$ plane that ends at critical point since we known that the transition is in a second order for $\mu_{B}=0$.

Another important question is the behavior of QCD in external magnetic fields. This is not a purely academic question since the properties of QCD in strong 
magnetic fields $B$ is relevant in several situations. For example, large magnetic fields exist inside ordinary neutron stars as well as magnetars [33]. In the latter case, the cores may be color superconducting and so it is important to study the effects of external magnetic fields in this phase. Similarly, it has been suggested that strong magnetic fields are created in heavy-ion collisions at the Relativistic Heavy-Ion Collider (RHIC) and the Large Hadron Collider (LHC) and that these play an important role 34]. In this case, the magnetic field strength has been estimated to be up to $B \sim 10^{19}$ Gauss, which corresponds to $|q B| \sim 6 m_{\pi}^{2}$, where $|q|$ is the charge of the pion. One of the interesting issues that has been raised is whether there is a splitting of the deconfinement and chiral transitions at finite $B$. This has spurred the interest in studying QCD in external fields. At zero baryon chemical potential this can be done from first principles using lattice simulations 35 37. At finite $\mu_{B}$ this is very difficult due to the infamous sign problem. Therefore one often resorts to effective theories that share some of the features of QCD, such as chiral symmetry breaking.

A low-energy effective theory that provides a systematic framework for systematic calculations is chiral perturbation theory. Chiral perturbation theory has been used to study the quark condensate in strong magnetic fields at zero temperature [38, 39], while the quark-hadron phase transition was investigated in Ref. [40]. The effects of external magnetic fields on the chiral transition have been studied in detail using the NJL model [41 50], the Polyakov-loop extended NJL model 51 53], the QM model [49, 50, 54], the (P)QM model [55], and the instanton-liquid model [56]. Similarly, the effects of strong magnetic field on the various color superconducting phases have been studied using the NJL model [57 61].

In this paper, we study the effects of magnetic fields on the chiral phase transition at finite temperature and finite baryon density. In order to do so, we employ the quark-meson model with two flavors coupled to a baryon chemical potential $\mu_{B}$. We consider the large $N_{c}$-limit which amounts to treat the sigma and pions at tree level. In this limit, we investigate the role of the fermionic vacuum fluctuations.

The paper is organized as follows. In Sec. II, we briefly discuss the QM model in an external magnetic field and at finite $\mu_{B}$. In Sec. III, we calculate the standard oneloop effective potential. In Sec. IV we discuss the thermodynamics and the numerical results in the large- $N_{c}$ limit. In Sec. V, we summarize and conclude.

\section{QUARK-MESON MODEL}

The Euclidean Lagrangian for the quark-meson model with $N_{f}=2$ flavors is

$$
\mathcal{L}=\mathcal{L}_{\text {meson }}+\mathcal{L}_{\text {quark }}+\mathcal{L}_{\text {Yukawa }}+\mathcal{L}_{\text {det }},
$$

where the various terms are

$$
\begin{aligned}
\mathcal{L}_{\text {meson }}= & \operatorname{Tr}\left[\partial_{\mu} \Phi^{\dagger} \partial_{\mu} \Phi\right]+m^{2} \operatorname{Tr}\left[\Phi^{\dagger} \Phi\right] \\
& +\frac{\lambda}{3} \operatorname{Tr}\left[\Phi^{\dagger} \Phi\right]^{2}-\frac{1}{2} h \operatorname{Tr}\left[\Phi+\Phi^{\dagger}\right], \\
\mathcal{L}_{\text {quark }}= & \bar{\psi}\left[\gamma_{\mu} \partial_{\mu}-\mu \gamma_{4}\right] \psi \\
\mathcal{L}_{\text {Yukawa }}= & g \bar{\psi}\left[\sigma-i \gamma_{5} \boldsymbol{\tau} \cdot \boldsymbol{\pi}\right] \psi, \\
\mathcal{L}_{\text {det }}= & c \operatorname{det}\left[\Phi+\Phi^{\dagger}\right],
\end{aligned}
$$

where

$$
\Phi=\frac{1}{2}(\sigma+\boldsymbol{\tau} \cdot \boldsymbol{\pi}) .
$$

Here, $\sigma$ is the sigma field, $\boldsymbol{\pi}$ denotes the neutral and charged pions. Moreover $\tau$ are the Pauli matrices, $\mu=$ $\frac{1}{2}\left(\mu_{u}+\mu_{d}\right)$ is the quark chemical potential, where $\mu_{u}$ and $\mu_{d}$ are the chemical potential for the $u$ and $d$ quarks, respectively. The baryon chemical potential is given by $\mu_{B}=3 \mu$. We set $\mu_{u}=\mu_{d}$ so that we are working at zero isospin chemical potential, $\mu_{I}=\frac{1}{2}\left(\mu_{u}-\mu_{d}\right)=0$. The Euclidean $\gamma$ matrices are given by $\gamma_{j}=i \gamma_{M}^{j}$ and $\gamma_{4}=\gamma_{M}^{0}$, where the index $M$ denotes Minkowski space. The fermion field is an isospin doublet

$$
\psi=\left(\begin{array}{l}
u \\
d
\end{array}\right) .
$$

If $h=0$, the first three terms in Eq. (1) are invariant un$\operatorname{der} U(2)_{L} \times U(2)_{R} \sim S U(2)_{L} \times S U(2)_{R} \times U(1)_{B} \times U(1)_{A}$. If $h \neq 0$, chiral symmetry is explicitly broken, otherwise it is spontaneously broken in the vacuum. Either way, the symmetry is reduced to $S U(2)_{V} \times U(1)_{B} \times U(1)_{A}$. Note that this requires $m^{2}<0$ which is assumed in the remainder of the paper. The $U(1)_{A}$ symmetry is also broken in the vacuum by instantons [62], and their effects are mimicked by the the determinant term Eq. (5). Since the $U(1)_{B}$ symmetry is always respected, symmetry of the QCD vacuum is $S U(2)_{V} \times U(1)_{B}$. In the following we set $c=0$ for simplicity.

Chiral symmetry is broken in the vacuum by a nonzero expectation value $v$ for the sigma field. We therefore make the replacement

$$
\sigma \rightarrow v+\tilde{\sigma},
$$

where $\tilde{\sigma}$ is a quantum fluctuating field with vanishing expecation value. After the shift (8), the tree-level potential is given by

$$
\mathcal{V}_{\text {tree }}=\frac{1}{2} m^{2} v^{2}+\frac{\lambda}{24} v^{4}-h v+\frac{1}{2} B^{2}+\mathcal{E}_{0},
$$


where the last term is the vacuum energy density . The tree-level masses for the sigma and neutral pion are

$$
\begin{aligned}
& m_{\sigma}^{2}=m^{2}+\frac{\lambda}{2} v^{2}, \\
& m_{\pi}^{2}=m^{2}+\frac{\lambda}{6} v^{2} .
\end{aligned}
$$

Note that the pion mass $m_{\pi}^{2}$ vanishes at the minimum of the tree-level potential in accordance with Goldstone's theorem. The charged pions $\pi^{ \pm}$and the quarks couple to the external magnetic field. This coupling is implemented by the substitution $\partial_{\mu} \rightarrow \partial_{\mu}+i q A_{\mu}$, where $A_{\mu}$ is the four-vector potential and $q$ is the electric charge of the particle. For a constant magnetic field $B$ in the $z$-direction, one can conveniently choose the four-vector potential as $\left(A_{0}, \mathbf{A}\right)=(0,0, B x, 0)$. The classical solutions to the Klein-Gordon equation in a constant magnetic field are well known and the dispersion relation is given by

$$
\left(E_{n, p_{z}}^{ \pm}\right)^{2}=p_{z}^{2}+m^{2}+\frac{1}{6} \lambda v^{2}+(2 n+1)|q B|
$$

where $n$ is an nonnegative integer, $q$ is the electric charge of the pion, and $p_{z}$ is the spatial momentum in the $z$-direction. The subscript \pm denotes $\pi^{ \pm}$and we note that the dispersion relations are identical. Similarly, the Dirac equation in a constant magnetic field $B$ can be solved straightforwardly and the dispersion relation for the quarks is given by

$$
E_{n, p_{z}}^{2}=p_{z}^{2}+m_{q}^{2}+(2 n+1-s)\left|q_{f} B\right|,
$$

where $m_{q}=g v$ is the quark mass after symmetry breaking, $q_{f}$ is the electric charge of the quark, and $s= \pm 1$ denote spin up/down, respectively.

\section{ONE-LOOP EFFECTIVE POTENTIAL}

In this section, we calculate the one-loop effective potential of the quark-meson model. This is done by taking into account the Gaussian fluctuations around the mean field $v$. The one-loop contribution $\mathcal{V}_{1}$ to the effective potential can be written as the sum of the contributions from the $\sigma$, pions, and quarks. This yields

$$
\mathcal{V}_{1}=\mathcal{V}_{\sigma}+\mathcal{V}_{\pi^{0}}+\mathcal{V}_{\pi^{+}}+\mathcal{V}_{\pi^{-}}+\mathcal{V}_{\mathrm{q}}
$$

where

$$
\begin{aligned}
\mathcal{V}_{\sigma} & =\frac{1}{2} \mathcal{F}_{P} \log \left[P^{2}+m_{\sigma}^{2}\right], \\
\mathcal{V}_{\pi^{0}} & =\frac{1}{2} \mathcal{F}_{P} \log \left[P^{2}+m_{\pi}^{2}\right],
\end{aligned}
$$

$$
\begin{aligned}
\mathcal{V}_{\pi^{ \pm}} & =\frac{1}{2} \frac{|q B| T}{2 \pi} \sum_{P_{0}, n} \int_{p_{z}} \log \left[P_{0}^{2}+p_{z}^{2}+M_{B}^{2}\right] \\
\mathcal{V}_{\mathrm{q}} & =-\sum_{f} \operatorname{Tr} \log \left[i \gamma_{\mu}\left(P_{\mu}+q_{f} A_{\mu}\right)+m_{q}-\mu \gamma_{4}\right]
\end{aligned}
$$

where $M_{B}^{2}=m_{\pi}^{2}+(2 n+1)|q B|$ and $f$ denotes the flavor. The symbol $\mathcal{V}_{P}$ is short-hand notation for

$$
\mathcal{f}_{P}=\left(\frac{e^{\gamma_{E}} \Lambda^{2}}{4 \pi}\right)^{\epsilon} T \sum_{P_{0}=2 \pi n T} \int_{p} \frac{d^{d} p}{(2 \pi)^{d}},
$$

where $d=3-2 \epsilon$ and $\Lambda$ is the renormalization scale associated with dimensional regularization in the $\overline{\mathrm{MS}}$ scheme. The integral over spatial momenta will be calculated in dimensional regularization. In the case of the charged pions, the sum-integral is replaced by a sum over Matsubara frequencies $P_{0}=2 \pi n T$, a sum over Landau levels $n$, and an integral over momenta in $d-2=1-2 \epsilon$ dimensions:

$$
\sum_{P} \rightarrow \frac{|q B| T}{2 \pi}\left(\frac{e^{\gamma_{E}} \Lambda^{2}}{4 \pi}\right)^{\epsilon} \sum_{P_{0}, n} \int_{p_{z}}
$$

where the prefactor $\frac{|q B|}{2 \pi}$ takes into account the degeneracy of the Landau levels and

$$
\int_{p_{z}}=\int \frac{d^{d-2} p_{z}}{(2 \pi)^{d-2}}
$$

Similarly, the contribution from the quarks can be written as

$$
\mathcal{V}_{q}=-N_{c} \sum_{\left\{P_{0}\right\}, n, s, f} \frac{\left|q_{f} B\right|}{2 \pi} \int_{p_{z}} \log \left[P_{0}^{2}+p_{z}^{2}+M_{q}^{2}\right]
$$

where $M_{q}^{2}=m_{q}^{2}+(2 n+1-s)\left|q_{f} B\right|, N_{c}$ is the number of colors, and the Matsubara frequencies are $P_{0}=(2 n+$ 1) $\pi T$.

The sum-integrals involving the contribution from the sigma and the neutral pion are essentially the same so we consider Eq. (15) with a general mass $M$. Summing over Matsubara frequencies we can write

$$
\begin{aligned}
& \mathcal{F}_{P} \log \left[P^{2}+M^{2}\right]=\int_{p}\left\{\sqrt{p^{2}+M^{2}}\right. \\
&\left.+2 T \log \left[1-e^{-\beta \sqrt{p^{2}+M^{2}}}\right]\right\} .
\end{aligned}
$$

The first term in Eq. (23) is ultraviolet divergent. Calculating it with dimensional regularization and expanding 
in powers of $\epsilon$ through order $\epsilon^{0}$, we obtain

$$
\begin{array}{r}
\oint_{P} \log \left[P^{2}+M^{2}\right]=-\frac{M^{4}}{32 \pi^{2}}\left(\frac{\Lambda^{2}}{M^{2}}\right)^{\epsilon}\left[\frac{1}{\epsilon}+\frac{3}{2}\right] \\
+\frac{T}{\pi^{2}} \int d p p^{2} \log \left[1-e^{-\beta \sqrt{p^{2}+M^{2}}}\right]
\end{array}
$$

We next consider the contribution to the effective potential from the charged pions. Summing over the Matsubara frequencies in Eq. (18), we obtain

$$
\begin{gathered}
\mathcal{V}_{\pi^{ \pm}}=\frac{|q B|}{4 \pi} \sum_{n} \int_{p_{z}}\left\{\sqrt{p_{z}^{2}+M_{B}^{2}}\right. \\
\left.+2 T \log \left[1-e^{-\beta \sqrt{p^{2}+M_{B}^{2}}}\right]\right\} .
\end{gathered}
$$

The first integral is ultraviolet divergent and we compute in dimensional regularization with $d=1-2 \epsilon$. This yields

$$
\int_{p_{z}} \sqrt{p_{z}^{2}+M_{B}^{2}}=-\frac{M_{B}^{2}}{4 \pi}\left(\frac{e^{\gamma_{E}} \Lambda^{2}}{M_{B}^{2}}\right)^{\epsilon} \Gamma(-1+\epsilon)
$$

Eq. (26) shows that the sum over Landau levels $n$ involves the term $M_{B}^{2-2 \epsilon}$. This sum is divergent for $\epsilon=0$ and we regulate it using zeta-function regularization. After scaling out a factor of $(2|q B|)^{1-\epsilon}$, this sum can be written as

$$
\begin{aligned}
\sum_{n} M_{B}^{2-2 \epsilon} & =(2|q B|)^{1-\epsilon} \sum_{n}\left[n+\frac{1}{2}+\frac{m_{\pi}^{2}}{2|q B|}\right]^{1-\epsilon} \\
& =(2|q B|)^{1-\epsilon} \zeta\left(-1+\epsilon, \frac{1}{2}+x\right)
\end{aligned}
$$

where $x=\frac{m_{\pi}^{2}}{2|q B|}$ and $\zeta(q, s)$ is the Hurwitz zeta function. The vacuum contribution then reduces to

$$
\mathcal{V}_{\pi^{ \pm}}^{\mathrm{vac}}=-\frac{(q B)^{2}}{8 \pi^{2}}\left(\frac{e^{\gamma_{E}} \Lambda^{2}}{2|q B|}\right)^{\epsilon} \Gamma(-1+\epsilon) \zeta\left(-1+\epsilon, \frac{1}{2}+x\right)
$$

Expanding Eq. (28), we obtain

$$
\mathcal{V}_{\pi^{ \pm}}^{\mathrm{vac}}=\frac{1}{64 \pi^{2}}\left(\frac{\Lambda^{2}}{2|q B|}\right)^{\epsilon}\left[\left(\frac{(q B)^{2}}{3}-m_{\pi}^{4}\right)\left(\frac{1}{\epsilon}+1\right)+8(q B)^{2} \zeta^{(1,0)}\left(-1, \frac{1}{2}+x\right)+\mathcal{O}(\epsilon)\right]
$$

where $\zeta^{(1,0)}\left(-1, \frac{1}{2}+x\right)$ is the derivative of the Hurwitz zeta function with respect to the first argument and where we have used that $\zeta\left(-1, \frac{1}{2}+x\right)=\frac{1}{24}-\frac{1}{2} x^{2}$.

The vacuum contributions from the quarks can be calculated in the same manner, and one finds [46, 63, 64]

$$
\begin{aligned}
\mathcal{V}_{q}^{\mathrm{vac}} & =\frac{N_{c}}{2 \pi^{2}} \sum_{f}\left(q_{f} B\right)^{2}\left(\frac{e^{\gamma_{E}} \Lambda^{2}}{2\left|q_{f} B\right|}\right)^{\epsilon} \Gamma(-1+\epsilon)\left[\zeta\left(-1+\epsilon, x_{f}\right)-\frac{1}{2} x_{f}^{1-\epsilon}\right] \\
& =\frac{N_{c}}{16 \pi^{2}} \sum_{f}\left(\frac{\Lambda^{2}}{2\left|q_{f} B\right|}\right)^{\epsilon}\left[\left(\frac{2\left(q_{f} B\right)^{2}}{3}+m_{q}^{4}\right)\left(\frac{1}{\epsilon}+1\right)-8\left(q_{f} B\right)^{2} \zeta^{(1,0)}\left(-1, x_{f}\right)-2\left|q_{f} B\right| m_{q}^{2} \log x_{f}+\mathcal{O}(\epsilon)\right]
\end{aligned}
$$

where $x_{f}=m_{q}^{2} / 2\left|q_{f} B\right|$. The divergences of the effective potential are given by Eqs. (24), (29), and (30). The divergences that depend on the magnetic field are given by

$$
\mathcal{V}_{1}^{\text {div }}=\frac{(q B)^{2}}{96 \pi^{2} \epsilon}+N_{c} \sum_{f} \frac{\left(q_{f} B\right)^{2}}{24 \pi^{2} \epsilon}
$$

These divergences are removed by wavefunction renormalization of the (external) gauge field $A_{\mu}$. This is done by making the replacement in the tree-level Lagrangian
Eq. (9) [65]:

$$
B^{2} \rightarrow B^{2}\left[1-\frac{q^{2}}{48 \pi^{2} \epsilon}-N_{c} \sum_{f} \frac{q_{f}^{2}}{12 \pi^{2} \epsilon}\right] .
$$

The remaining divergences in $\mathcal{V}_{1}$ are given by

$$
\mathcal{V}_{1}^{\text {div }}=-\frac{1}{64 \pi^{2} \epsilon}\left[m_{\sigma}^{4}+3 m_{\pi}^{4}-4 N_{c} N_{f} m_{q}^{4}\right]
$$

These are the same divergences as one encounters in vanishing magnetic field and so the usual renormalization procedure can be used to eliminate them. This is done 
by adding a vacuum energy counterterm $\Delta \mathcal{E}_{0}$ and making the replacements $m^{2} \rightarrow m^{2}+\delta m^{2}$ and $\lambda \rightarrow \lambda+\delta \lambda$ in the tree-level effective potential (9), where

$$
\begin{aligned}
\Delta \mathcal{E}_{0} & =\frac{m^{4}}{16 \pi^{2} \epsilon}, \\
\delta m^{2} & =\frac{\lambda m^{2}}{16 \pi^{2} \epsilon},
\end{aligned}
$$

$$
\delta \lambda=\frac{\lambda^{2}}{8 \pi^{2} \epsilon}-\frac{3 N_{c} N_{f} g^{4}}{2 \pi^{2} \epsilon} .
$$

The renormalized one-loop contribution to the effective potential at $T=0$ then becomes

$$
\begin{aligned}
\mathcal{V}_{1}^{\mathrm{vac}}= & -\frac{m_{\sigma}^{4}}{64 \pi^{2}}\left[\log \frac{\Lambda^{2}}{m_{\sigma}^{2}}+\frac{3}{2}\right]-\frac{m_{\pi}^{4}}{64 \pi^{2}}\left[\log \frac{\Lambda^{2}}{m_{\pi}^{2}}+\frac{3}{2}\right]-\frac{m_{\pi}^{4}}{32 \pi^{2}}\left[\log \frac{\Lambda^{2}}{2|q B|}+1\right]+\frac{N_{c} m_{q}^{4}}{16 \pi^{2}} \sum_{f}\left[\log \frac{\Lambda^{2}}{2\left|q_{f} B\right|}+1\right] \\
& +\frac{(q B)^{2}}{4 \pi^{2}} \zeta^{(1,0)}\left(-1, \frac{1}{2}+x\right)-\frac{N_{c}}{2 \pi^{2}} \sum_{f}\left(q_{f} B\right)^{2} \zeta^{(1,0)}\left(-1, x_{f}\right)-\frac{N_{c} m_{q}^{2}}{8 \pi^{2}} \sum_{f}\left|q_{f} B\right| \log \frac{m_{q}^{2}}{2\left|q_{f} B\right|} \\
& +\frac{(q B)^{2}}{96 \pi^{2}} \log \frac{\Lambda^{2}}{2|q B|}+N_{c} \sum_{f} \frac{\left(q_{f} B\right)^{2}}{24 \pi^{2}} \log \frac{\Lambda^{2}}{2\left|q_{f} B\right|} .
\end{aligned}
$$

The finite-temperature contribution $\mathcal{V}_{1}^{T, \mu}$ to the effective potential is given by

$$
\begin{aligned}
\mathcal{V}_{1}^{T, \mu}= & \frac{T}{2 \pi^{2}} \int_{0}^{\infty} d p p^{2} \log \left[1-e^{-\beta \sqrt{p^{2}+m_{\sigma}^{2}}}\right]+\frac{T}{2 \pi^{2}} \int_{0}^{\infty} d p p^{2} \log \left[1-e^{-\beta \sqrt{p^{2}+m_{\pi}^{2}}}\right] \\
& +\frac{|q B| T}{\pi^{2}} \sum_{n} \int_{0}^{\infty} d p \log \left[1-e^{-\beta \sqrt{p^{2}+M_{B}^{2}}}\right]-N_{c} \sum_{s, f, n} \frac{\left|q_{f} B\right| T}{2 \pi^{2}} \int_{0}^{\infty} d p \log \left[1+e^{-\beta\left(\sqrt{p^{2}+M_{q}^{2}} \pm \mu\right)}\right]
\end{aligned}
$$

The full one-loop effective potential is then given by the sum of Eqs. (9), (36), and (37). The renormalized vacuum energy $\mathcal{E}_{0}$, background field $B$, mass $m$, and quartic coupling $\lambda$ satisfy the renormalization group equations

$$
\begin{aligned}
\Lambda \frac{d \mathcal{E}_{0}}{d \Lambda} & =\frac{m^{4} \lambda}{8 \pi^{2}} \\
\Lambda \frac{d B^{2}}{d \Lambda} & =-\frac{(q B)^{2}}{24 \pi^{2}}-N_{c} \sum_{f} \frac{\left(q_{f} B\right)^{2}}{6 \pi^{2}} \\
\Lambda \frac{d m^{2}}{d \Lambda} & =\frac{m^{2} \lambda}{8 \pi^{2}} \\
\Lambda \frac{d \lambda}{d \Lambda} & =\frac{\lambda^{2}}{16 \pi^{2}}-\frac{3 N_{c} N_{f} g^{4}}{\pi^{2}} .
\end{aligned}
$$

Using the renormalization-group equations, we conclude that the one-loop effective potential is renormalization group invariant.

We close this section by taking the limit $B \rightarrow 0$ in Eqs. (36) and (37). We then need the large- $x$ behavior of $\zeta^{(1,0)}\left(-1, \frac{1}{2}+x\right)$ and $\zeta^{(1,0)}\left(-1, x_{f}\right)$. These are given by

$$
\begin{aligned}
\zeta^{(1,0)}\left(-1, \frac{1}{2}+x\right) & =\frac{1}{2} x^{2}\left(\log x-\frac{1}{2}\right)+\ldots, \\
\zeta^{(1,0)}\left(-1, x_{f}\right) & =\frac{1}{2} x_{f}^{2}\left(\log x_{f}-\frac{1}{2}\right)-\frac{1}{2} x_{f} \log x_{f}+\ldots
\end{aligned}
$$

Inserting the expansions (42) and (43) into (36), we obtain the standard one-loop vacuum term

$$
\begin{aligned}
\mathcal{V}_{1}^{\mathrm{vac}}= & -\frac{m_{\sigma}^{4}}{64 \pi^{2}}\left[\log \frac{\Lambda^{2}}{m_{\sigma}^{2}}+\frac{3}{2}\right]-\frac{3 m_{\pi}^{4}}{64 \pi^{2}}\left[\log \frac{\Lambda^{2}}{m_{\pi}^{2}}+\frac{3}{2}\right] \\
& +\frac{N_{c} N_{f} m_{q}^{4}}{16 \pi^{2}}\left[\log \frac{\Lambda^{2}}{m_{q}^{2}}+\frac{3}{2}\right] .
\end{aligned}
$$

In Eq. (37), we change variable $p_{\perp}^{2}=2|q B| n$, which yields $p_{\perp} d p_{\perp}=|q B| d n$. Replacing the sum by integrals, we obtain 


$$
\begin{aligned}
\mathcal{V}_{1}^{T, \mu}= & \frac{T}{2 \pi^{2}} \int_{0}^{\infty} d p p^{2} \log \left[1-e^{-\beta \sqrt{p^{2}+m_{\sigma}^{2}}}\right]+\frac{3 T}{2 \pi^{2}} \int_{0}^{\infty} d p p^{2} \log \left[1-e^{-\beta \sqrt{p^{2}+m_{\pi}^{2}}}\right] \\
& -\frac{N_{c} N_{f} T}{\pi^{2}} \int_{0}^{\infty} d p p^{2} \log \left[1+e^{-\beta\left(\sqrt{p^{2}+m_{q}^{2}} \pm \mu\right)}\right] .
\end{aligned}
$$

The full one-loop effective potential at $B=0$ is the sum of Eqs. (91), (44) and (45)).

\section{THERMODYNAMICS AND NUMERICAL RESULTS}

In the previous section, we calculated the one-loop effective potential at finite temperature $T$ and finite baryon chemical potential $\mu_{B}$. A common approximation in the QM model is to neglect the quantum and thermal fluctuations of the mesons, which is equivalent to the large- $N_{c}$ limit. One hopes that the contributions from the quarks include the most important effects [4, 54]. In this paper, we will apply this approximation and defer the use of more sophisticated methods to a subsequent paper [66]. The one-loop effective potential then reduces to

$$
\begin{aligned}
\mathcal{V}_{0+1}= & \frac{1}{2} m^{2} v^{2}+\frac{\lambda}{24} v^{4}-h v+\frac{N_{c} m_{q}^{4}}{16 \pi^{2}} \sum_{f}\left[\log \frac{\Lambda^{2}}{2\left|q_{f} B\right|}+1\right]-\frac{N_{c}}{2 \pi^{2}} \sum_{f}\left(q_{f} B\right)^{2} \zeta^{(1,0)}\left(-1, x_{f}\right) \\
& -\frac{N_{c} m_{q}^{2}}{8 \pi^{2}} \sum_{f}\left|q_{f} B\right| \log \frac{m_{q}^{2}}{2\left|q_{f} B\right|}-N_{c} \sum_{s, f, n} \frac{\left|q_{f} B\right| T}{2 \pi^{2}} \int_{0}^{\infty} d p \log \left[1+e^{-\beta\left(\sqrt{p^{2}+M_{q}^{2}} \pm \mu\right)}\right],
\end{aligned}
$$

where we for simplicity have omitted all $v$-independent terms. Notice that the renormalized effective potential Eq. (46) for large values of the order parameter $v$ is unbounded from below due to the dominant term $-N_{c} m_{q}^{4} \log \left(m_{q}^{2} / 2 q_{f} B\right) / 16 \pi^{2}$ that arises from $\zeta^{(1,0)}\left(-1, x_{f}\right)$ at large $x_{f}$. The unboundedness of the fermionic functional determinant is well known for $B=$ 0 and arises here as well. It is due to the minus sign in Eq. (18). However, it is stabilized by the bosonic contributions in Eq. (44) for realistic values of the coupling $\lambda$, (cf. Eqs. (49)- (52) and the numerical values given below). Generally, the reliability of perturbative (vacuum) calculations and the stability of the effective potential are nontrivial issues, see e. g. for a thorough discussion [67 69].

Let us first briefly discuss magnetic catalysis of dynamical symmetry breaking, namely the effect that chiral symmetry is broken dynamically for any nonzero magnetic field when it is intact for $B=0$. This effect is now well established, see e. g. [63, 70 75]. In the present case, chiral symmetry at vanishing magnetic field $B$ corresponds to choosing $m^{2}>0{ }^{1}$. The minimum of the effective potential is found by minimizing the effective potential (46) with $\mu_{B}=T=h=0$. In the limit $B \rightarrow 0$, we know that the expectation value $v$ is exponentially small [63] and so we can expand $\mathcal{V}_{0+1}$ around $v=0$. This yields ${ }^{2}$

$$
\begin{aligned}
\frac{d \mathcal{V}_{0+1}}{d v} & \approx v\left[m^{2}+\frac{N_{c} g^{2}}{8 \pi^{2}} \sum_{f}\left|q_{f} B\right| \log \frac{\pi m_{q}^{2}}{\left|q_{f} B\right|}\right] \\
& =0 .
\end{aligned}
$$

Either $v=0$ or we obtain $v^{2}$

$$
v^{2}=\frac{2^{\frac{2}{3}}|q B|}{3 g^{2} \pi} \exp \left[-\frac{8 \pi^{2} m^{2}}{N_{c} g^{2}|q B|}\right] .
$$

The nontrivial solution Eq. (48) corresponds to the local minimum of $\mathcal{V}_{0+1}$. Eq. (48) has the same functional form

${ }^{1} \mathrm{~m}^{2}>0$ corresponds to a symmetric vacuum state at tree level. In the NJL model, symmetry breaking is always a loop effect which takes place for $G$ smaller than a critical coupling $G_{c}$.

2 We have used $\zeta^{(1,0)}\left(-1, x_{f}\right)=$ const $-x_{f} \log \sqrt{2 \pi} x_{f}+\ldots$ for small values of $x_{f}$. 
with the replacement $1 / G \rightarrow m^{2} / g^{2}$ as in the NJL-model calculations of e. g. Ref. 63]. The reason is that we are basically evaluating the same fermionic functional determinant although we are using a different ultraviolet regulator $\Lambda$.

We next discuss the determination of the parameters of the Lagrangian (11). In the vacuum, we have $\mu_{B}=0$ and $f_{\pi}=v$, where $f_{\pi}$ is the pion decay constant. At tree level, the mass parameter $m^{2}$, the couplings $\lambda$ and $g$, and the symmetry breaking parameter $h$ can be expressed in terms of observable masses of the sigma and pions, and the pion decay constant $f_{\pi}$ :

$$
\begin{aligned}
m^{2} & =-\frac{1}{2}\left(m_{\sigma}^{2}-3 m_{\pi}^{2}\right) \\
\lambda & =\frac{3\left(m_{\sigma}^{2}-m_{\pi}^{2}\right)}{f_{\pi}^{2}} \\
g & =\frac{m_{q}}{f_{\pi}} \\
h & =f_{\pi} m_{\pi}^{2}
\end{aligned}
$$

We use a sigma mass of $m_{\sigma}=800 \mathrm{MeV}$, a constituent quark mass of $m_{q}=300 \mathrm{MeV}$, and a pion decay constant of $f_{\pi}=93 \mathrm{MeV}$. In the chiral limit, $h=0$, this yields $m^{2}=-320000 \mathrm{MeV}^{2}, \lambda=222$, and $g=3.2258$. At the physical point, we obtain $m^{2}=-291018 \mathrm{MeV}^{2}, \lambda=$ 215.29, and $g=3.2258$. In the remainder of the paper, we set $N_{c}=3$ and $c=0$.

We first consider the effective potential at $T=\mu=$ 0 . As noted above, we have determined the parameters of the theory at tree level. Quantum fluctuations, i. e. loop corrections, will modify the classical potential and it depends on the renormalization scale $\Lambda$. We can choose $\Lambda$ such that the minimum of the one-loop effective potential in the vacuum (for $B=0$ ) still is at $v=f_{\pi}$. This is done by requiring

$$
\left.\frac{d \mathcal{V}_{0+1}}{d v}\right|_{v=f_{\pi}}=0
$$

This is equivalent to requiring that the one-loop selfenergy of the pion at zero external momentum vanish, $\Pi_{1, \pi}(0)=0$. From Eq. (44), this yields

$$
\left[\log \frac{\Lambda^{2}}{g^{2} f_{\pi}^{2}}+1\right]=0,
$$

whose solution is $\Lambda=181.96 \mathrm{MeV}$. We will use this value in the remainder of the paper.

In Fig. 1, we plot the tree-level potential (solid curve) as well as the one-loop effective potential in the chiral limit for $|q B|=0,|q B|=5 m_{\pi}^{2}$, and $|q B|=10 m_{\pi}^{2}$ with $T=\mu=0$ The dashed curve is for $|q B|=0$, the dotted curve is $|q B|=5 m_{\pi}^{2}$ and the dash-dotted curve is for $|q B|=10 m_{\pi}^{2}$. We notice that the potential becomes deeper with increasing magnetic field and that the minimum moves to larger values of $v^{3}$. Thus the magnetic field enhances symmetry breaking. This is in agreement with earlier findings [54, 55, 63, 70 75]. We also note

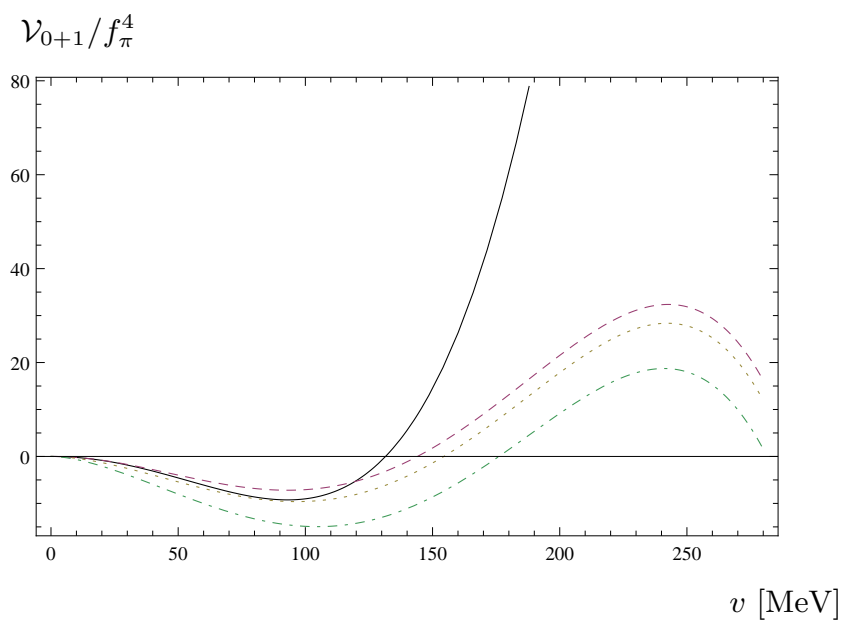

FIG. 1: Normalized effective potential $\mathcal{V}_{0+1} / f_{\pi}^{4}$ in the chrial limit for $T=\mu_{B}=0$. Tree level (solid curve), one-loop with $|q B|=0$ (dashed curve), one loop with $|q B|=5 m_{\pi}^{2}$ (dotted curve), and one loop with $|q B|=10 m_{\pi}^{2}$ (dash-dotted curve).

that the local maximum of the effective potential for large values of $v$ becomes lower with increasing magnetic field. Thus for sufficiently strong magnetic fields, the local minimum at $v=93 \mathrm{MeV}$ ceases to exist and the system has no longer a metastable state. This is the instability that we discussed above. This in disagreement with Ref. [55]. In their work, the authors subtract the fermionic vacuum fluctuations at $B=0$. Thus they subtract a $v$-dependent contribution given by the last term in Eq. (44) which leads to a one-loop effective potential that is independent of the renormalization scale $\Lambda$ and is stable for large values of $v$.

It was mentioned in the introduction that the inclusion of the fermion vacuum fluctuation term can change the order of the phase transition in the chiral limit as well as strongly influence physical observable. This was discussed in detail for $\mu_{B}=0$ in Ref. 7]. It turns out that this is the case in the entire $T-\mu_{B}$ phase diagram for $B=0[10]$. We find that this is also the case in a strong magnetic field. In Fig. 2, we show the normalized effective potential with no vacuum fluctuations for three different values of the baryon chemical potential $\mu_{B}$ and

3 Note, however, that the one-loop effective potential for $|q B|=0$ is more shallow than the tree-level potential. 
$T=0$ as a function of $v$. Clearly, the transition is first order if the vacuum fluctuations are not included.

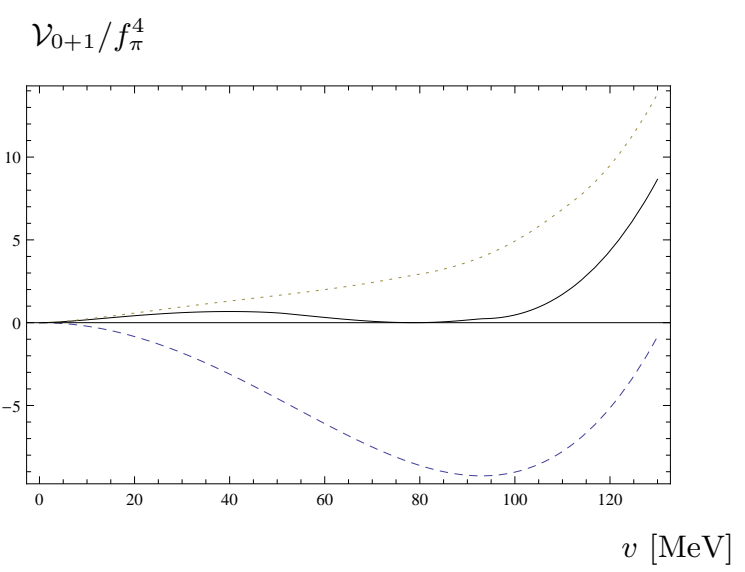

FIG. 2: Normalized effective potential $\mathcal{V}_{0+1} / f_{\pi}^{4}$ without vacuum fluctuations for $T=0$ and $|q B|=5 \mathrm{~m}_{\pi}^{2}$ as a function of $v$ for three different values of $\mu: \mu=0$ (dashed curve), $\mu=\mu_{c}=301.6 \mathrm{MeV}$ (solid curve), and $\mu=358 \mathrm{MeV}$ (dotted curve).

Similarly, in Fig. 3. we show the normalized effective potential with vacuum fluctuations for three different values of the baryon chemical potential $\mu_{B}$ and $T=0$ as a function of $v$. The transition is second order if the vacuum fluctuations are included.

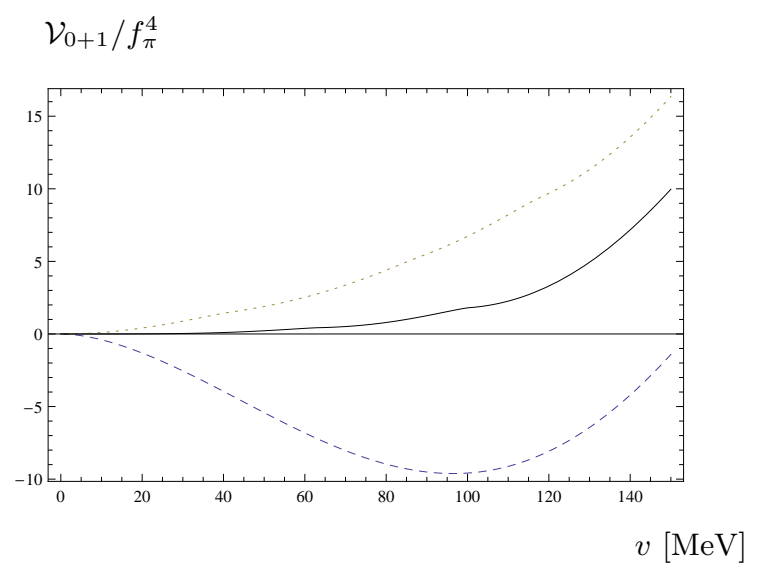

FIG. 3: Normalized effective potential $\mathcal{V}_{0+1} / f_{\pi}^{4}$ including vacuum fluctuations for $T=0$ and $|q B|=5 m_{\pi}^{2}$ as a function of $v$ for three different values of $\mu$ : $\mu=0$ (dashed curve), $\mu=\mu_{c}=324 \mathrm{MeV}$ (solid curve), and $\mu=380 \mathrm{MeV}$ (dotted curve).

In Figs. 45, we show the phase diagram as a function of $\mu_{B}$ and $T$ in the chiral limit. In Eq. (46), there is a sum over Landau levels and for each value of $\mu_{B}$, we include sufficiently many terms so that our result for $T_{c}$ is converging. For example, for $\mu_{B}=0$, we must typically sum the first ten term in the series, while for $T=0$ it suffices to include the first few terms in the series.

In Fig 4, we have included the fermionic vacuum fluctuations and the dashed curve indicates a second-order transition. In the case where we include the quantum fluctuations, the critical temperature for $\mu=0$ is $T_{c}=179 \mathrm{MeV}$ and the critical chemical potential for $T=0$ is $\mu_{c}=324 \mathrm{MeV}$. In comparison, the critical temperature at vanishing magnetic field is $T_{c}=176 \mathrm{MeV}$, the critical chemical potential is $\mu_{c}=321 \mathrm{MeV}$, and the phase transition is also second order for all values of $\mu$ [10]. Calculating the critical temperature for $\mu_{B}=0$ and $|q B|=10 m_{\pi}^{2}$ yields $T_{c}=184 \mathrm{MeV}$ and so it seems to be increasing very weakly with the strength of the magnetic field.

In Fig [5, we have omitted the vacuum term and the solid curve indicates a first-order transition. In the case where the quantum fluctuations are omitted, the critical temperature for $\mu=0$ is $T_{c}=160 \mathrm{MeV}$ and the critical chemical potential for $T=0$ is $\mu_{c}=301.6 \mathrm{MeV}$. In comparison, the critical temperature at vanishing magnetic field is $T_{c}=179 \mathrm{MeV}$, the critical chemical potential is $\mu_{c}=347 \mathrm{MeV}$, and the phase transition is also first order for all values of $\mu$ [10]. In contrast to the zero- $B$ case, the exclusion of the vacuum fluctuation decreases the critical temperature significantly.

In Ref. [10], it was shown using optimized perturbation theory [11] that the inclusion of the vacuum and thermal contributions from the bosons significantly lowers the temperature of the transition. Whether this implies that including the effects of the bosons will lower the critical temperature for nonzero $B$ as well is not known. In particular, the $B$-dependence of the critical temperature is an open question, see also the discussion below.

There have been a number of model calculations using chiral perturbation theory, the NJL model, the NJL model coupled to the Polyakov loop, the QM model, and the Polyakov-loop extended model. Except for chiral perturbation theory [40], they all predict an increase of the critical temperature with increasing magnetic field. This is in disagreement with the lattice simulations of Ref. 37] which show a significant decrease of the crossover temperature.

In Ref. [40], the authors are using chiral perturbation theory to investigate the quark-hadron phase transition as a function of the magnetic field at the physical point. They compare the pressure in the hadronic phase with that of the quark-gluon plasma phase for an ideal gas of quarks and gluons, and subtracting the vacuum en- 


\section{$T[\mathrm{MeV}]$}

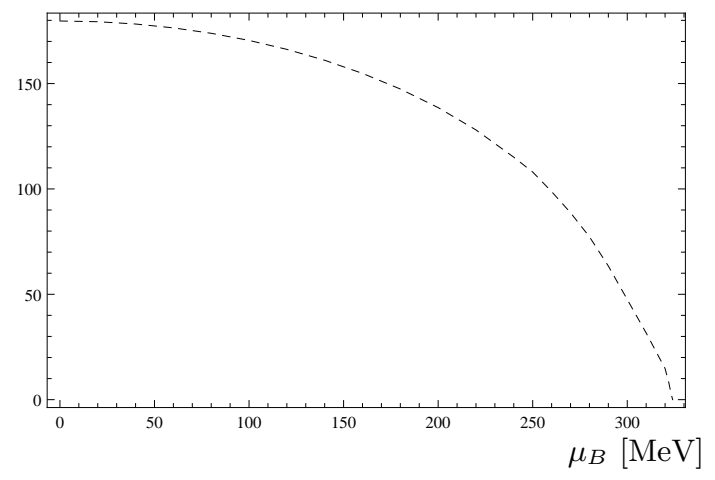

FIG. 4: Phase diagram in the $\mu_{B}-T$ plane for $|q B|=5 m_{\pi}^{2}$ in the chiral limit. Fermionic vacuum fluctuations are included.

\section{$T[\mathrm{MeV}]$}

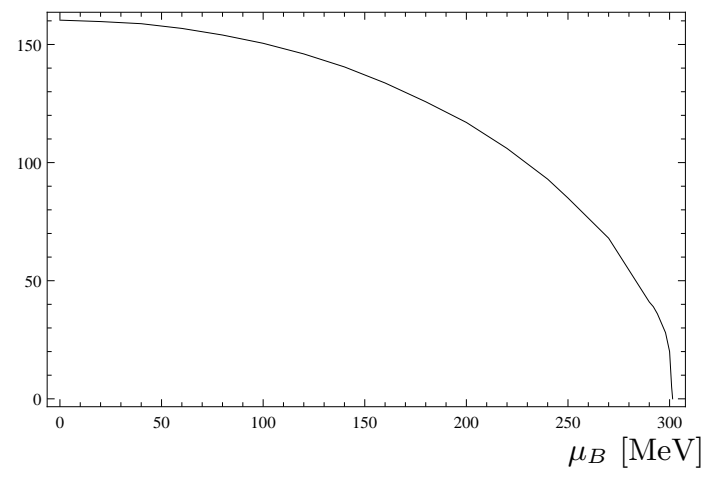

FIG. 5: Phase diagram in the $\mu_{B}-T$ plane for $|q B|=5 m_{\pi}^{2}$ in the chiral limit. Fermionic vacuum fluctuations are excluded.

ergy due to a nonzero gluon condensate $\left\langle g^{2} G_{\mu \nu} G^{\mu \nu}\right\rangle$. For weak magnetic fields, the transition is first order. The line of first-order transitions ends at critical point $(\sqrt{|q B|}, T)=(600,104) \mathrm{MeV}$. For larger values of $|q B|$, the transition is a crossover. The authors of Ref. [55] report that they find a first-order transition at the physical point if they keep the remaining $B$-dependent vacuum fluctuations and a crossover if they are ignored. Our sample calculations at the physical point for $|q B|=5 m_{\pi}^{2}$ (not shown) suggest that the transition is a crossover (for $\left.\mu_{B}=0\right)$. This is in agreement with the lattice simulations in Ref. [37]. The disagreement between Ref. 55] and the present results can probably be traced back to a different treatment of the vacuum fluctuations, as discussed earlier. The disagreement between various approaches deserves further investigation.

\section{SUMMARY AND OUTLOOK}

In the present work, we have calculated the one-loop effective potential for the quark-meson model at finite temperature and baryon density in an external magnetic field. We have made the common approximation where we ignore all quantum and thermal effects of the bosons and hence treat them at tree level. We have seen that the critical temperature and the order of the phase transition depend on whether one includes the vacuum fluctuations. Taking a model seriously means including the effects of all its degrees of freedom. It therefore questionable to throw out some terms unless one can show that they are not important. For example, the effective potential is receiving contributions from vacuum fluctuations from all energies scales up to the ultraviolet cutoff $\Lambda$ of the effective theory. This is the region of validity of the low-energy effective theory and it is therefore not obvious that they can be omitted.

Given the conflicting results of various model calculations and lattice calculations, clearly more work needs to be done. On the analytical side, an important extension is to include the vacuum and thermal effects of the bosons. The dominant contributions at weak coupling from the bosons arise from the daisy or ring diagrams. There has been some work on resumming the ring diagrams in the presence of a magnetic field. For example, the authors of Ref. 76] investigated their role in the electroweak phase transition in the standard model. Similarly, their effects at weak fields have been studied in Ref. [77] in the context of the chiral transition. One should apply sophisticated resummation techniques or nonperturbative methods such as optimized perturbation theory [11], the 2PI effective action formalism [78], or the functional renormalization group [79]. These methods are known to correctly predict a second-order phase transition for $\mu_{B}=B=0[9,80,81$.

\section{Acknowledgments}

The authors would like to thank E. S. Fraga for useful discussions. R. Khan was supported by the Higher Education Commision of Pakistan (HEC).
[1] M. A. Stephanov, Prog. Theor. Phys.Suppl. 153, 139 (2000), Int. J. Mod. Phys. A 20, 4387 (2005).
[2] M. G. Alford, A. Schmitt, K. Rajagopal, and T. Schafer, Rev. Mod. Phys. 80, 1455 (2008). 
[3] H. C. G. Caldas, A. L. Mota, and M. C. Nemes, Phys Rev. D 63, 056011 (2001).

[4] O. Scavenius, A. Mocsy, I. N. Mishustin, and D. H. Rischke. Phys. Rev. C 64, 045202 (2001).

[5] A. Mocsy, I. N. Mishustin, and P. J. Ellis, Phys. Rev. C 70, 015204 (2004).

[6] E. S. Bowman and J. I. Kapusta. Phys. Rev. C 79, 015202 (2009).

[7] V. Skokov, B. Friman, E. Nakano, K. Redlich, and B.-J. Schaefer Phys. Rev. D 82, 034029 (2010).

[8] P. Kovacs and Zs. Szep. Phys. Rev. D 75, 025015 (2007).

[9] B.-J. Schaefer and J. Wambach, Nucl. Phys. A 757, 479 (2005); Phys. Rev. D 75, 085015 (2007).

[10] J. O. Andersen, R. Khan, and L. T. Kyllingstad, e-Print: arXiv:1102.2779 [hep-ph].

[11] S. Chiku and T. Hatsuda, Phys. Rev. D 58, 076001 (1998).

[12] C. S. Fischer, J. Luecker, J. A. Mueller, Phys. Lett. B 702, 438 (2011).

[13] K. Fukushima, Phys. Lett. B 591, 277 (2004).

[14] C. Ratti, M. A. Thaler, and W. Weise, Phys. Rev. D 73, 014019 (2006).

[15] B.-J. Schaefer, J. M. Pawlowski, and J. Wambach, Phys. Rev. D 76, 074023 (2007).

[16] T. Kähärä and K. Tuominen, Phys. Rev. D 78, 034015 (2008); ibid 82, 114026 (2010).

[17] E. Nakano, B.-J. Schaefer, B. Stokic, B. Friman, and K. Redlich, Phys. Lett. B 682, 401 (2010).

[18] V. Skokov, B. Stokic, B. Friman, and K. Redlich, Phys. Rev. C 82, 015206 (2010).

[19] V. Skokov, B. Friman, and K. Redlich, Phys. Rev. C 83, 054904 (2011).

[20] G. Marko and Zs. Szep, Phys. Rev. D 82, 065021 (2010); e-Print: arXiv:1012.5855 [hep-ph].

[21] T. K. Herbst, J. M. Pawlowski, and B.-J. Schaefer, Phys. Lett. B 696, 58 (2011).

[22] U. S. Gupta and V. K. Tiwari, e-Print: arXiv:1107.1312

[23] H. Abuki, M. Ciminale, R. Gatto, N. D. Ippolito, G. Nardulli, and M. Ruggieri, Phys. Rev. D 78, 014002 (2008). field.

[24] S. Roessner, T. Hell, C. Ratti, and W. Weise, Nucl. Phys. A 814, 118 (2008).

[25] T. Hell, S. Rossner, M. Cristoforetti, and W. Weise, Phys. Rev. D 79, 014022 (2009).

[26] A. E. Radzhabov, D. Blaschke, M. Buballa, and M. K. Volkov Phys. Rev. D 83, 116004 (2011).

[27] D. Horvatic, D. Blaschke, D. Klabucar, and O. Kaczmarek, Phys. Rev. D 84, 016005 (2011).

[28] T. Sasaki, Y. Sakai, H. Kouno, and Masanobu Yahiro, Phys. Rev. D 82, 116004 (2010); e-Print: arXiv:1104.2394 [hep-ph].

[29] F. Xu, H. Mao, T. K. Mukherjee, and M. Huang, Phys. Rev. D 84, 074009 (2011).

[30] R. D. Pisarski and F. Wilczek, Phys. Rev. D 29, 338, (1984).

[31] S. D. H. Hsu and M. Schwetz, Phys. Lett. B 432, 203 (1998).

[32] M. Buballa, Phys. Rept. 407, 205 (2005).
[33] J. M. Lattimer and M. Prakash Phys. Rept. 442, 109 (2007).

[34] D. E. Kharzeev, L. D. McLerran, and H. J. Warringa, Nucl. Phys. A 803, 227 (2008).

[35] M. D'Elia, S. Mukherjee, and F. Sanfilippo, Phys. Rev. D 82, 051501(R) (2010).

[36] M. D'Elia and F. Negro, Phys. Rev D83, 114028 (2011)

[37] G. S. Bali, F. Bruckmann, G. Endrodi, Z. Fodor, S. D. Katz, S. Krieg, A. Schafer, and K. K. Szabo, e-Print: arXiv:1111.4956 [hep-lat].

[38] I. A. Shuspanov and A. V. Smilga, Phys. Lett. B 402, 351 (1997).

[39] T. D. Cohen, D. A. McGady, and E. S. Werbos, Phys. Rev. C 76, 055201 (2007).

[40] N. O. Agasian and S. M. Fedorov, Phys. Lett. B 663, 445 (2008).

[41] S. P. Klevansky and R. H. Lemmer, Phys. Rev. D 39, 3478 (1989).

[42] V. P. Gusynin, V. A. Miransky, and I.A. Shovkovy, Phys. Lett. B 349, 477 (1995); Nucl. Phys. B 462, 249 (1996).

[43] A. Yu. Babansky, E. V. Gorbar, and G. V. Shchepanyuk, Phys. Lett. B 419, 272 (1998).

[44] D. Ebert and K. G. Klimenko, Nucl. Phys. A 728, 203 (2003).

[45] B. Hiller, A. A. Osipov, A. H. Blin, and J. da Providencia, SIGMA 4024 (2008).

[46] J. K. Boomsma and D. Boer, Phys. Rev. D 81, 074005 (2010).

[47] B. Chatterjee, H. Mishra, and A. Mishra, Phys. Rev. D 84, 14016 (2011)

[48] S. S. Avancini, D. P. Menezes, and C. Providencia, Phys. Rev. C 83, 065805 (2011).

[49] M. Frasca and M. Ruggieri, Pys. Rev. D 83, 094024 (2011).

[50] A. Rabhi and C. Providencia, Phys. Rev. C 83, 055801 (2011).

[51] R. Gatto and M. Ruggieri, Phys. Rev. D 82, 054027 (2010); ibid 83034016 (2011).

[52] K. Kashiwa, Phys. Rev. D 83, 117901 (2011).

[53] A. J. Mizher, E. S. Fraga, and M. N. Chernodub, PoS FACESQCD, 020 (2010).

[54] E. S. Fraga and A. J. Mizher, Phys. Rev. D. 78, 025016 (2008).

[55] A. J. Mizher, M. N. Chernodub, and E. S. Fraga, Phys. Rev. D 82, 105016 (2010).

[56] S.-Il. Nam and C.-W. Kao, Phys. Rev. D 83, 096009 (2011).

[57] E. J. Ferrer, V. de la Incera, and C. Manuel, Phys. Rev. Lett. 95, 152002 (2005), Nucl. Phys B 74788 (2006).

[58] E. J. Ferrer and V. de la Incera. Phys. Rev. D 76, 045011 (2007).

[59] K. Fukushima and H. J. Warringa, Phys. Rev. Lett. 100, 032007 (2008).

[60] J. L. Noronha and I. A. Shovkovy, Phys. Rev. D 76 105030 (2007).

[61] S. Fayazbakhsh and N. Sadooghi, Phys. Rev. D 82, 045010 (2010); ibid 83, 025026 (2011).

[62] G. 't Hooft Phys. Rep. 142, 357 (1986).

[63] D. Ebert, K. G. Klimenko, M. A. Vdovichenko, and A. S. 
Vshivtsev, Phys. Rev. D 61, 025005 (1999).

[64] D. P. Menezes, M. B. Pinto, S. S. Avancini, A. P. Martinez and C. Providencia, Phys. Rev. C 79, 035807 (2009); D. P. Menezes, M. B. Pinto, S. S. Avancini, and C. Providencia, Phys. Rev. C 80, 065805 (2009).

[65] P. Elmfors, D. Persson, and B.-S. Skagerstam, Astropart.Phys. 2, 5299 (1994).

[66] A. Amador and J. O. Andersen, in preparation.

[67] M. Sher, Phys. Rept. 179, 273 (1989).

[68] C. Ford, D. R. T. Jones, P. W. Stephenson, and M. B. Einhorn, Nucl. Phys. B 395, 17 (1993).

[69] M. B. Einhorn and D. R. T. Jones, JHEP 0704, 051 (2007).

[70] K.G. Klimenko, Z. Phys. C 54, 323 (1992).

[71] V. P. Gusynin, V. A. Miransky, and I. A. Shovkovy, Phys. Rev. Lett. 73, 3499 (1994).

[72] V. P. Gusynin, V.A. Miransky, and I.A. Shovkovy, Nucl. Phys. B462, 249 (1996).

[73] C. N. Leung, Y. J. Ng, and A. W. Ackley, Phys. Rev. D
54, 4181 (1996); ibid D 55, 6504 (1997).

[74] C.N. Leung, S.-Y. Wang, Nuclear Physics B 747, 266 (2006).

[75] E. J. Ferrer and V. de la Incera, Phys. Lett. B, 481, 287 (2000); Nuclear Physics B 824, 217 (2010).

[76] V. Skalozub and M. Bordag, Int. J. Mod. Phys. A 15, 349 (2000); V. Skalozub and V. Demchik, e-Print: hep-th/9912071.

[77] A. Ayala, A. Sanchez, G. Piccinelli, and S. Sahu, Phys. Rev. D 71, 023004 (2005); A. Ayala, A. Bashir, A. Raya, and A. Sanchez, Phys. Rev. D 80, 036005 (2009).

[78] J. M. Cornwall, R. Jackiw, and E. Tomboulis, Phys. Rev. D 10, 2428 (1974).

[79] C. Wetterich, Nucl. Phys. B. 352, 529 (1991).

[80] S. Chiku, Prog. Theor. Phys. 104, 1129 (2000).

[81] A. Arrizabalaga and U. Reinosa, Nucl. Phys. A 785, 234 (2007). 